\section{Cistectomía parcial laparoscópica asistida por robot en un paciente con paraganglioma vesical}

Sánchez-Aquino U, ${ }^{1}$ Trujillo-Ortiz L, ${ }^{1}$ Sedano-Basilio J, ${ }^{1}$ Gómez-Sánchez J, ${ }^{1}$ Herrera-Muñoz J, ${ }^{1}$ Preciado-Estrella D, ${ }^{1}$ y colaboradores.

\section{Resumen}

ANTECEDENTES: el paraganglioma vesical es un tumor raro, que se origina del tejido embrionario cromafín de los nervios simpáticos asociados con la pared vesical. Este tipo de tumor puede ser funcionante o no funcionante, según la secreción de catecolaminas.

CASO CLÍNICO: hombre de 61 años de edad, que acudió al servicio médico por hematuria macroscópica, formadora de coágulos amorfos, asociada con síntomas urinarios irritativos, palpitaciones y cefalea durante la micción. La urotomografía mostró un tumor de $2.5 \mathrm{~cm}$ en el domo vesical. Se realizó la resección transuretral de un tumor de la de vejiga con reporte histopatológico de paraganglioma vesical. Los estudios hormonales resultaron negativos y no se encontró evidencia de metástasis en los estudios de extensión. Se llevó a cabo una cistectomía parcial laparoscópica asistida por robot de forma exitosa. Actualmente el paciente se encuentra en seguimiento, sin evidencia de recurrencia.

CONCLUSIONES: los paragangliomas vesicales son tumores raros, que aparecen en menos de $0.05 \%$ de todas las neoplasias vesicales y en menos de $1 \%$ de todos los feocromocitomas. En la vía urinaria los sitios más comunes de manifestación son: la vejiga $(79.2 \%)$ y la uretra $(12.7 \%)$. Las manifestaciones clínicas son variables, pero se ha descrito principalmente cefalea y palpitaciones asociadas con la micción. En pacientes con paragangliomas localizados se indica cirugía y en quienes padecen tumores recurrentes y metastásicos se prefiere tratamiento paliativo. Las manifestaciones de los paragangliomas vesicales son extremadamente variables, por lo que se requiere una alta sospecha en los pacientes con hipertensión y hematuria. El tratamiento de elección consiste en la cistectomía parcial.

PALABRAS CLAVE: paraganglioma, vejiga, feocromocitoma.

Rev Mex Urol. 2017 Sep-Oct;77(5):393-400.

\section{Robotic-assisted laparoscopic partial cystectomy in a patient with bladder paraganglioma}

Sánchez-Aquino U, ${ }^{1}$ Trujillo-Ortiz L, ${ }^{1}$ Sedano-Basilio J, ${ }^{1}$ Gómez-Sánchez J, ${ }^{1}$ Herrera-Muñoz J, ${ }^{1}$ Preciado-Estrella $D,{ }^{1}$ y colaboradores.

\section{Abstract}

BACKGROUND: Bladder paraganglioma is a rare tumor originating in the chromaffin embryonic tissue of the sympathetic nerves associat-

\footnotetext{
${ }^{1}$ Residente de Urología.

${ }^{2}$ Residente de Patología.

${ }^{3}$ Adscrito al servicio de Patología.

${ }^{4}$ Jefe del servicio de Patología.

${ }^{5}$ Adscrito al servicio de Urología

${ }^{6}$ Jefe del servicio de Urología

Hospital General Dr. Manuel Gea González, Ciudad de México.
}

Recibido: diciembre 2016

Aceptado: julio 2017

Correspondencia

Dr. Ulises Cristóbal Sánchez-Aquino

ulisescsa@gmail.com

Este artículo debe citarse como

Sánchez-Aquino U, Trujillo-Ortiz L, Sedano-Basilio J, Gómez-Sánchez J, Herrera-Muñoz J, PreciadoEstrella D, y col. Cistectomía parcial laparoscópica asistida por robot en un paciente con paraganglioma vesical. Rev Mex Urol. 2017 sep-oct;77(5):393-400. DOI: https://doi.org/10.24245/revmexurol.v77i5.1107 
ed with the bladder wall. They can be functioning or non-functioning, depending on catecholamine secretion.

CLINICAL CASE: A 61-year-old man sought medical attention for amorphous clot-forming gross hematuria associated with irritative urinary symptoms, palpitations, and headache during micturition. A computed tomography urogram revealed a $2.5-\mathrm{cm}$ tumor in the bladder dome. Transurethral resection of the bladder tumor was performed and the outcome of the histopathologic study was bladder paraganglioma. Hormonal testing was negative and extension studies showed no evidence of metastasis. The patient successfully underwent robotic-assisted laparoscopic partial cystectomy. He is currently in follow-up, with no signs of recurrence.

CONCLUSIONS: Bladder paraganglioma is a rare tumor representing less than $0.05 \%$ of all bladder tumors and less than $1 \%$ of all pheochromocytomas. The bladder is the most common presentation site in the urinary tract $(79.2 \%)$, followed by the urethra $(12.7 \%)$. Clinical presentation varies, consisting mainly of headache and palpitations upon micturition. Surgery is the preferred treatment for localized paragangliomas, whereas palliative therapy is preferred for recurrent and metastatic tumors. Becuase of the extremely varied presentation of bladder paraganglioma, a high degree of suspicion is necessary in the evaluation of patients with high blood pressure and hematuria. The treatment of choice is partial cystectomy.

KEYWORDS: Paraganglioma; Bladder; Pheochromocytoma

\author{
Residente de Urología. \\ ${ }^{2}$ Residente de Patología. \\ ${ }^{3}$ Adscrito al servicio de Patología. \\ ${ }^{4}$ Jefe del servicio de Patología. \\ ${ }^{5}$ Adscrito al servicio de Urología \\ ${ }^{6}$ Jefe del servicio de Urología \\ Hospital General Dr. Manuel Gea González, \\ Ciudad de México. \\ Correspondence \\ Dr. Ulises Cristóbal Sánchez-Aquino \\ ulisescsa@gmail.com
}

\section{ANTECEDENTES}

Los paragangliomas son tumores neuroendocrinos que suelen originarse en las células cromafines. Se clasifican según la secreción de catecolaminas y en el caso de los productores de hormonas se asocian con síntomas inespecíficos, como cefalea y palpitaciones, y en algunos casos hipertensión paroxística, síncope urinario y hematuria. ${ }^{1}$

Los paragangliomas localizados en la médula adrenal se denominan feocromocitomas. Aparecen en menos de $0.05 \%$ de todos los tumores vesicales y en menos de $1 \%$ de todos los feocromocitomas. En el aparato genitourinario son poco frecuentes; sin embargo, la vejiga es el sitio más común de manifestación (79.2\%), seguido de la uretra $(12.7 \%)$, pelvis $(4.9 \%)$ y uréter $(3.2 \%)$. Mantienen una relación de 3:1 entre mujeres y hombres, y suelen aparecer entre la segunda y tercera décadas de la vida. ${ }^{2}$

Los síntomas varían desde ataques de cefalea asociada con la micción y palpitaciones, incluso síntomas inespecíficos, como parestesias y disnea; sin embargo, $17 \%$ de los paragangliomas vesicales no son hormonalmente activos, lo que dificulta establecer el diagnóstico. ${ }^{3}$ La mayor parte de los paragangliomas asintomáticos solo pueden diagnosticarse mediante estudios histopatológicos. ${ }^{4}$ 
Entre 5 y $15 \%$ de los paragangliomas vesicales son malignos; ${ }^{5}$ sin embargo, no se han establecido criterios histológicos para distinguir entre tumores benignos y malignos. La invasión local o metástasis a distancia confirman que el tumor es maligno. ${ }^{6}$

El tratamiento de elección consiste en resección quirúrgica, incluida la resección transuretral, cistectomía parcial o cistectomía total. ${ }^{7}$ Con el avance en las técnicas de laparoscopia, la cistectomía parcial se ha convertido en el tratamiento de elección. ${ }^{8}$ La técnica laparoscópica asistida por robot ha permitido efectuar una disección más precisa, además de los beneficios ya establecidos del abordaje laparoscópico como: estancia hospitalaria, tiempo de recuperación y sangrado menores.

El seguimiento es necesario para detectar recurrencias tardías. Deben realizarse estudios de imagen, orina y cistoscopia de forma regular, aunque no se ha establecido un consenso para la frecuencia de los mismos. ${ }^{9}$

\section{CASO CLÍNICO}

Paciente de 61 años de edad, sin antecedentes de relevancia para el padecimiento actual, quien acudió al servicio médico por hematuria macroscópica, formadora de coágulos amorfos, intermitente, asociada con síntomas urinarios irritativos, ansiedad, palpitaciones y mareo durante la micción.

Durante la exploración física se reportó tensión arterial de 130-80 mmHg, sin datos patológicos adicionales. Se realizaron estudios hormonales, con determinación de catecolaminas (adrenalina, noradrenalina y dopamina), metanefrinas (metanefrina y normetanefrina) y ácido vanililmandélico en orina de 24 horas, que se encontraron dentro de los parámetros normales. El gamagrama con metayodobencilguanidina
(MIBG) mostró hipercaptación vesical, sin localizar tejido cromafin en otros sitios.

En la urotomografía se observó una lesión en la pared vesical anterior de $2.5 \mathrm{~cm}$. Se efectuó cistoscopia con resección transuretral del tumor vesical en forma parcial (Figura 1). El reporte de patología fue paraganglioma vesical, por lo que se llevó a cabo cistectomía parcial laparoscópica asistida por robot.

Con el efecto de la anestesia general, se colocó al paciente en posición de litotomía y se efectuó cistoscopia delimitando con energía monopolar el margen quirúrgico. De forma simultánea se inició el neumoperitoneo y el procedimiento laparoscópico asistido por robot, además de realizar la disección del espacio extraperitoneal, desinsertando el ligamento umbilical medio. Después de la disección vesical, mediante transiluminación (Figura 2) por cistoscopia se guió el corte peritumoral, dejando un margen de $2 \mathrm{~cm}$ a la lesión (Figura 3), la cistectomía fue parcial (Figura 4) y a continuación se reparó la vejiga en dos planos con sutura V-Lock (Figura 5). El paciente tuvo evolución satisfactoria, por lo que egresó dos días después del posoperatorio y se le retiró la sonda Foley a los 7 días. Del procedimiento quirúrgico se obtuvo una tumoración de consistencia ahulada de $2.5 \mathrm{~cm}$ (Figura 6) y el reporte histopatológico confirmó la histología, mediante estudios de inmunohistoquímica, con márgenes quirúrgicos libres de la neoplasia.

\section{DISCUSIÓN}

El primer caso de paraganglioma vesical fue reportado en 1953 por Zimmerman. ${ }^{5}$ Aún se desconoce el mecanismo por el que se originan estos tumores. La mayor parte de los paragangliomas son esporádicos y cerca de $10 \%$ se asocian con alteraciones genéticas como: paraganglioma familiar, neurofibromatosis tipo 1, enfermedad de Von Hippel-Lindau y neoplasia endocrina 


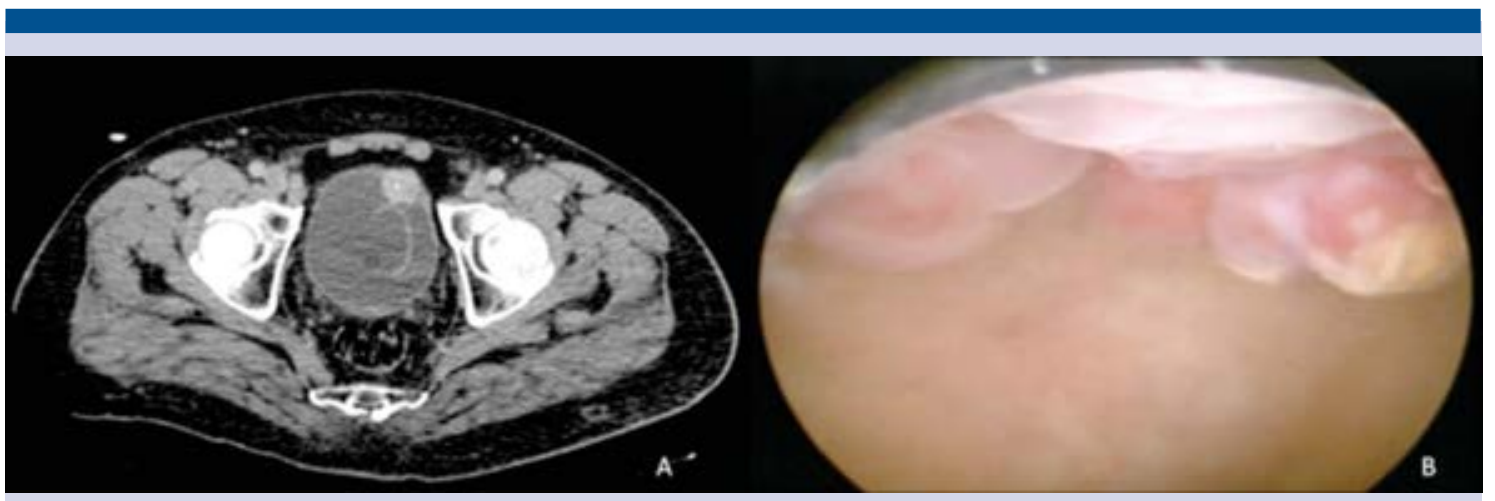

Figura 1. Tomografía (A) y cistoscopia (B) que evidencian una lesión tumoral en la pared vesical anterior de $2.5 \mathrm{~cm}$.

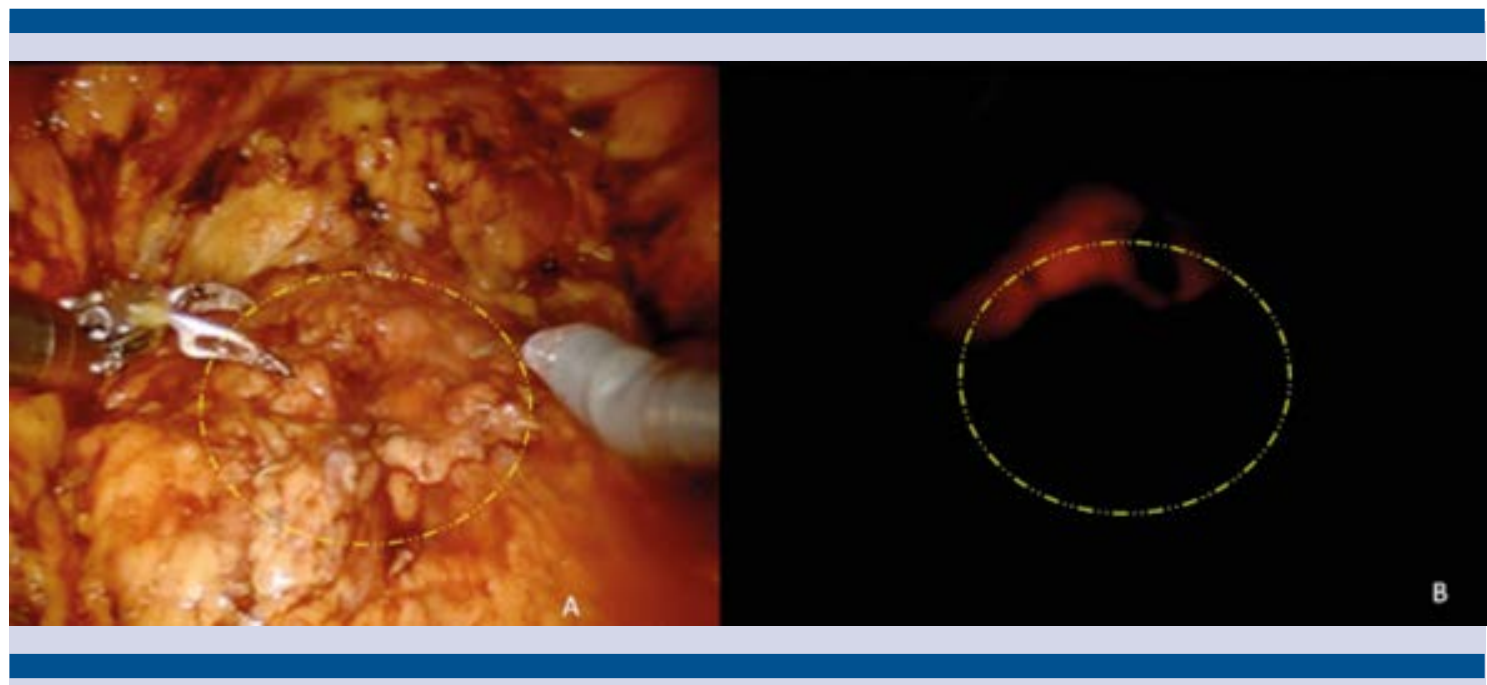

Figura 2. Transiluminación para marcaje de bordes quirúrgicos tumorales.

múltiple tipo $2 .^{10}$ Se ha reportado mayor afección en mujeres que en hombres. ${ }^{11}$ El sitio más común de manifestación es en la vía urinaria, principalmente en la vejiga $(79.2 \%)$, y la mayor parte $(83 \%)$ son activos hormonalmente. ${ }^{12}$ De ahí que la sospecha de un paraganglioma debe conducir a pruebas que incluyan la determinación de catecolaminas y ácido vanililmandélico en orina de 24 horas. ${ }^{13}$ En el paciente de este estudio, la historia clínica no sugería alteraciones endocrinas que guiaran hacia un paraganglioma funcionante, por lo que el abordaje inicial fue por hematuria, encontrándose en la urotomografía el tumor vesical ya comentado. La localización preoperatoria es extremadamente importante para confirmar el diagnóstico. La tomografía permite evaluar la relación entre el tumor y la mucosa muscular y los tejidos perivesicales. ${ }^{14} \mathrm{La}$ metayodobencilguanidina (MIBG) es un análogo de la norepinefrina, que se absorbe por el tejido del paraganglioma y se utiliza para establecer el diagnóstico y localización de los paragangliomas 


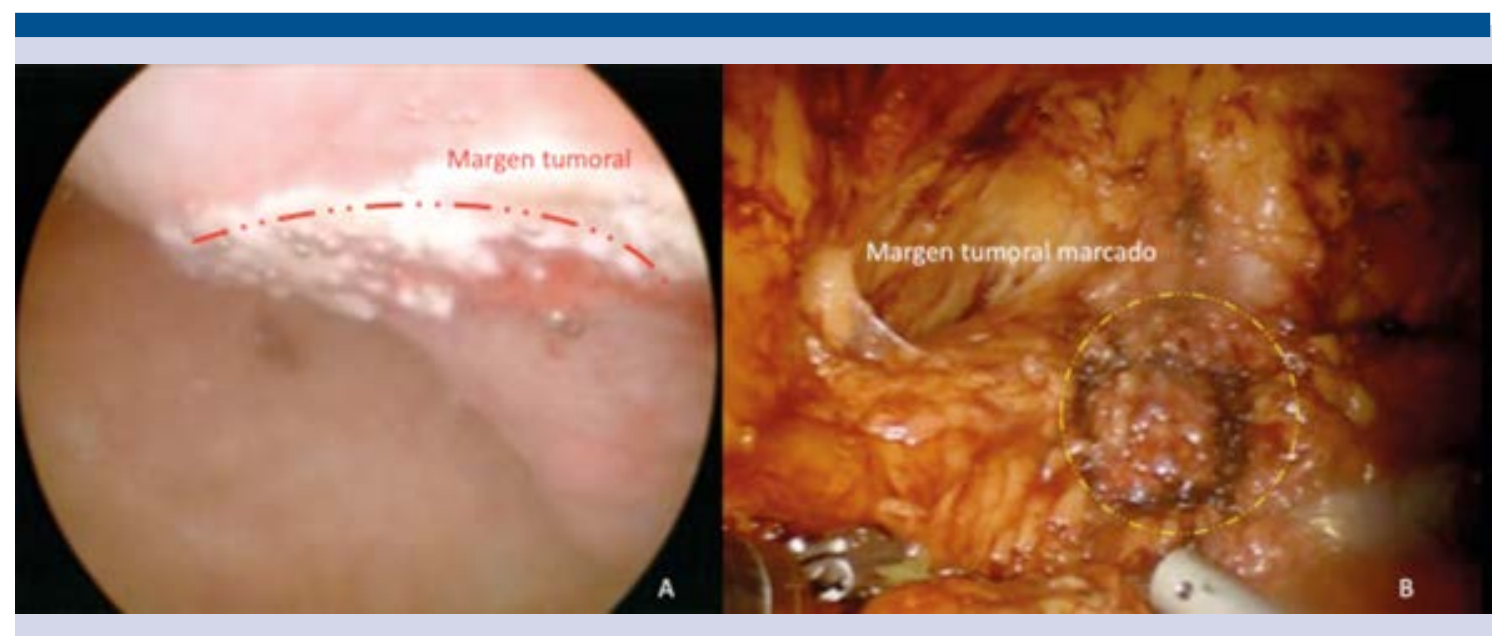

Figura 3. Delimitación del borde quirúrgico tumoral con energía monopolar por cistoscopia (A) y laparoscopia (B).

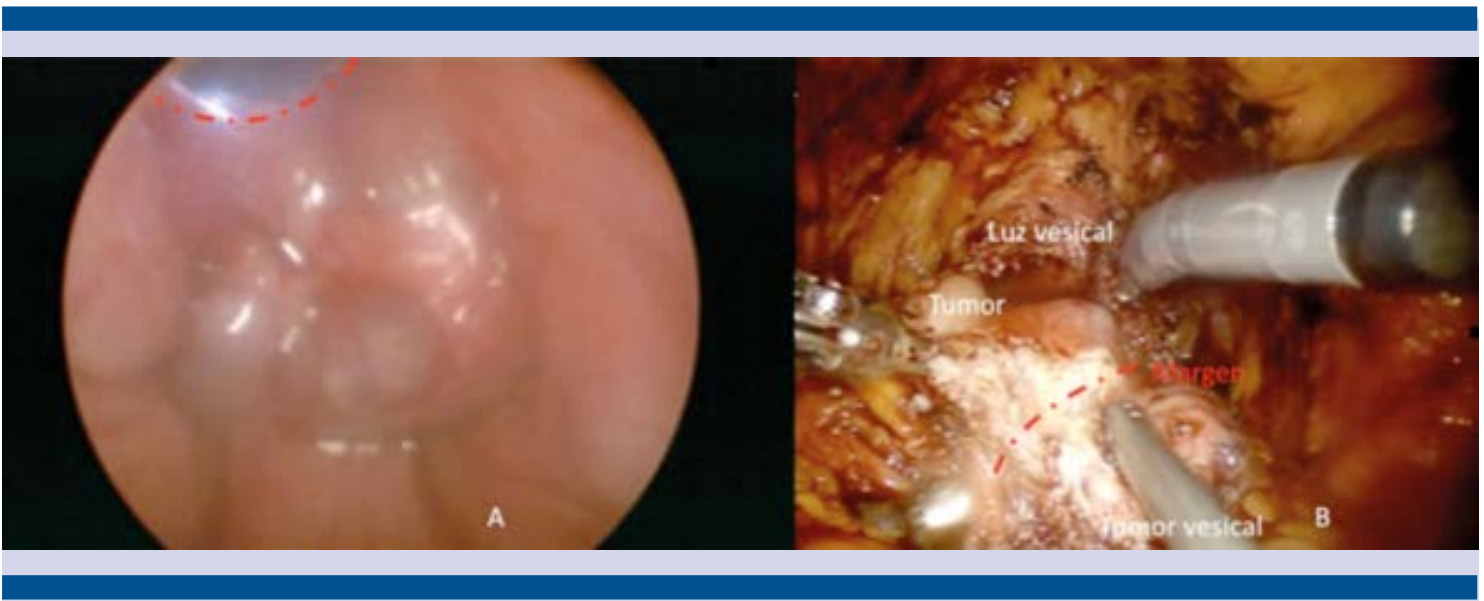

Figura 4. Apertura de la luz vesical con visión cistoscópica (A) y laparoscópica (B).

extraadrenales, con una especificidad cercana a $100 \%$ y sensibilidad de $90 \%$. La tomografía por emisión de positrones ofrece aún más precisión que el gamagrama con MIBG para localizar los paragangliomas, debido a su resolución espacial más alta. ${ }^{15}$

La apariencia durante la cistoscopia es de una masa que protruye a la luz vesical, bien vascularizada, que muestra la mucosa continua e intacta; sin embargo, en pacientes con tumores funcionales, la cistoscopia induce fluctuaciones de la presión sanguínea, por lo que su uso es limitado y no se recomienda la biopsia de la tumoración por el riesgo de provocar crisis hipertensivas y sangrado, debido a su alta vascularización. ${ }^{16}$ En nuestro caso, el diagnóstico al momento de la cistoscopia no se había establecido, por lo que se realizó la resección transuretral del mismo. 


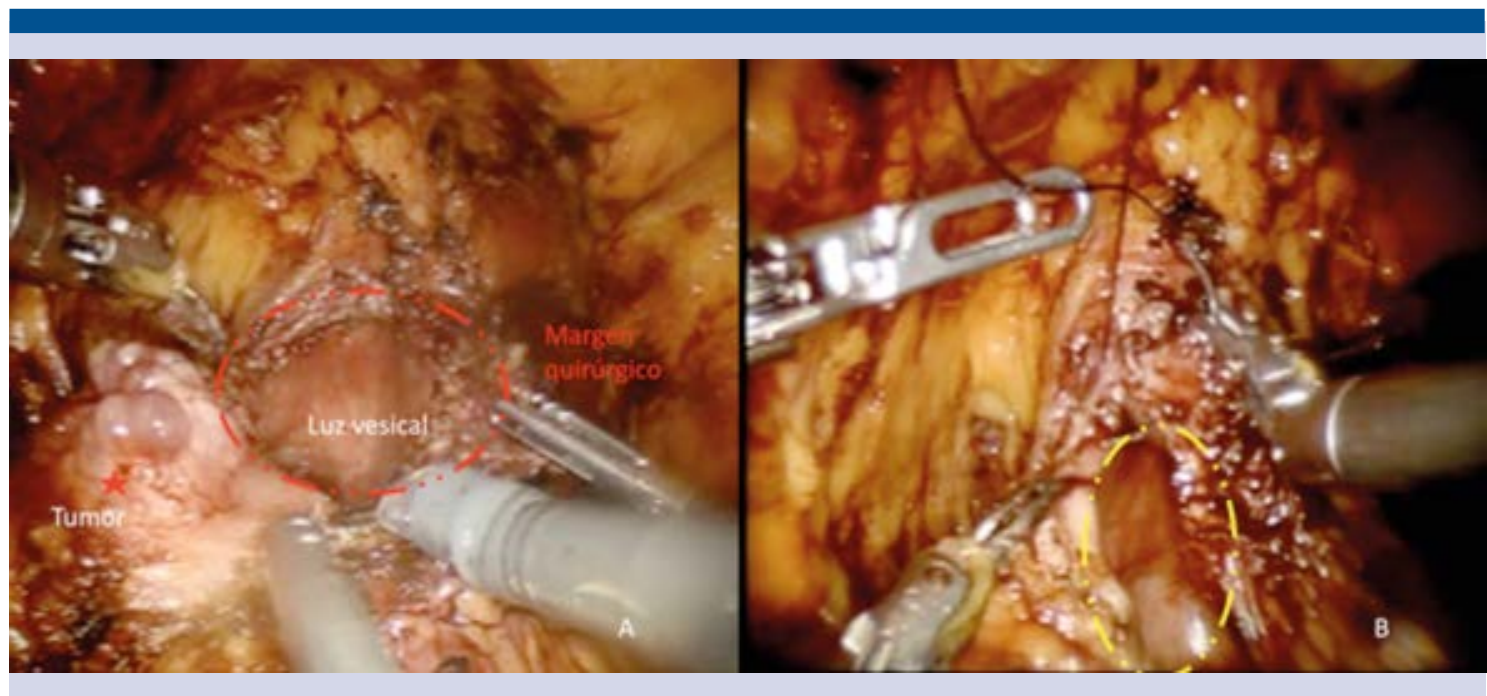

Figura 5. Resección del tumor vesical a punto de ser terminada (A) y cierre en dos planos (B).

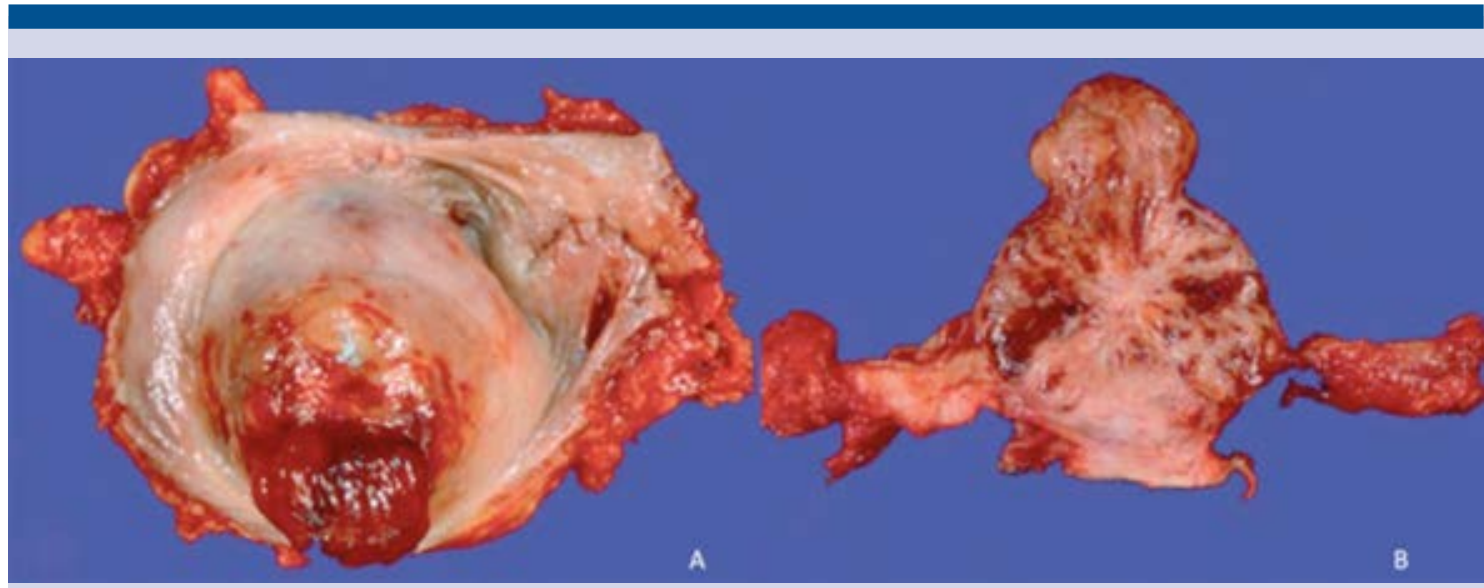

Figura 6. Imagen macroscópica (A) y al corte (B) de la pieza quirúrgica.

El diagnóstico de la mayor parte de los paragangliomas se establece mediante estudio histopatológico. Los tumores muestran características similares a los feocromocitomas adrenales y, en ocasiones, se requieren estudios de inmunohistoquímica para efectuar el diagnóstico definitivo. ${ }^{17}$
El tratamiento de elección es la intervención quirúrgica del tumor y la preparación preoperatoria es fundamental en pacientes con características de hipertensión paroxística durante la micción. Es necesario estabilizar la presión antes de efectuar la cirugía, así como la administración de agentes alfa bloqueadores o bloqueadores 


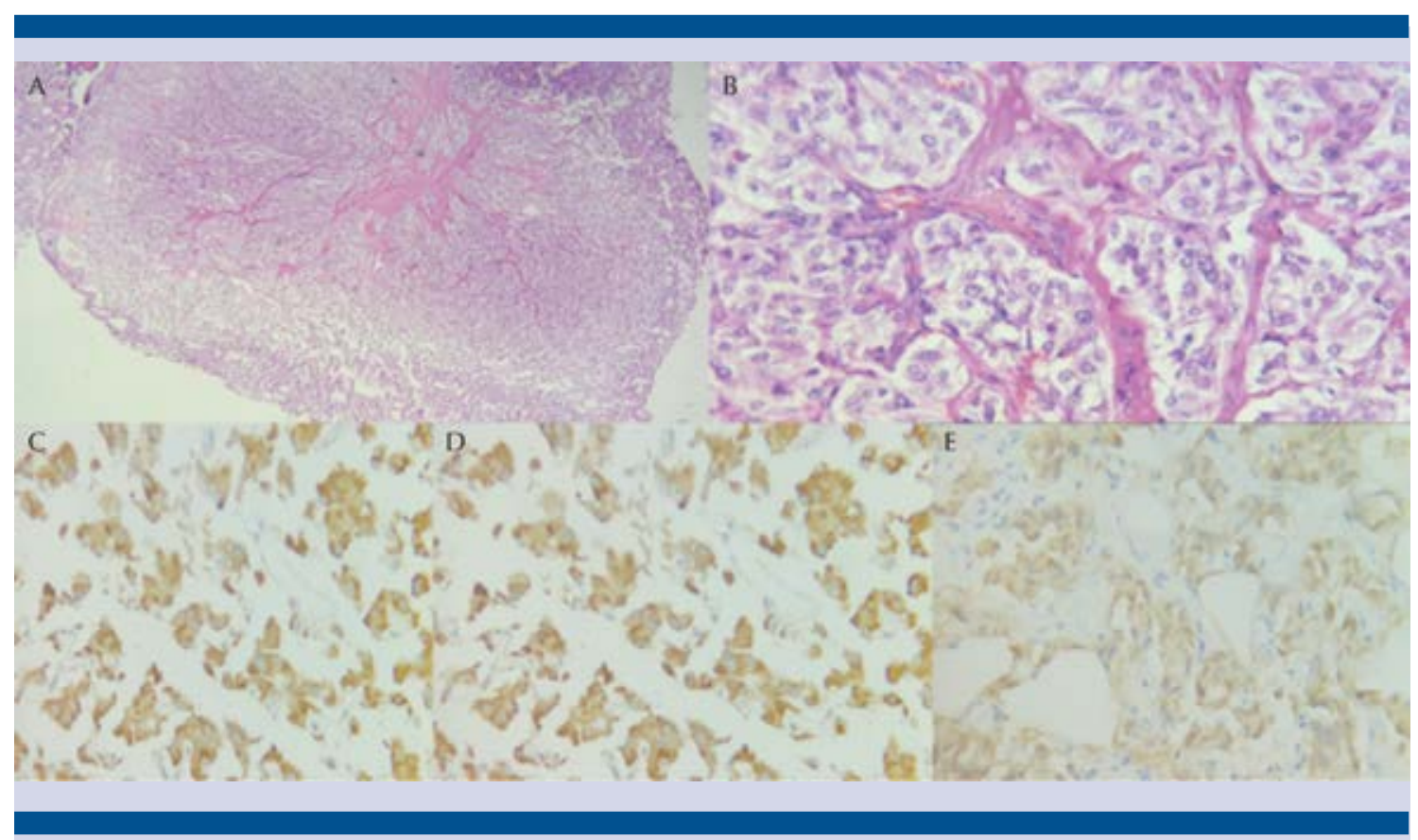

Figura 7. Apariencia histológica en nidos a 10X (A) y 100X (B) típica del paraganglioma; inmunohistoquímica para cromogranina $(C)$, sinaptofisina (D) y S-100 (E) positivas.

de los canales de calcio durante dos semanas, con la finalidad de inhibir la liberación de catecolaminas. $^{18}$

Con los avances en las técnicas de laparoscopia, la cistectomía parcial laparoscópica se ha convertido en el tratamiento de elección. ${ }^{19} \mathrm{La}$ resección transuretral es factible en pacientes con tumores menores de 2 a $3 \mathrm{~cm}$, sin infiltración parietal..$^{20}$ En el presente caso, se contó con la posibilidad de efectuar la cirugía laparoscópica asistida por robot, debido a las ventajas para la disección, así como las propias de un procedimiento laparoscópico, con adecuada evolución posoperatoria, sin evidencia de recurrencia hasta el momento.

\section{CONCLUSIONES}

Los paragangliomas vesicales suelen manifestarse de forma variable, por lo que se requiere una alta sospecha diagnóstica. El paraganglioma vesical que no produce hormonas dificulta aún más el diagnóstico, pues la manifestación clínica no aporta datos adicionales. En los pacientes con paraganglioma vesical sin producción de hormonas es importante efectuar el estudio histopatológico, además de los estudios de extensión adecuados. El tratamiento de elección consiste en cistectomía parcial; en el paciente de este estudio se llevó a cabo cistectomía parcial laparoscópica asistida por robot.

\section{Financiamiento}

No existió financiamiento de ninguna organización pública o privada.

\section{Conflicto de interés}

Los autores declaran no tener conflicto de interés. 


\section{REFERENCIAS}

1. Kappers $M H$, van den Meiracker $A H$, Alwani RA, Kats $E$, Baggen MG. Paraganglioma of the urinary bladder. Neth J Med 2008;66:163-165.

2. Lai $Y, C$ Chen $D, Y u Z$, et al. Non-functioning paraganglioma of the urinary bladder: A case report and review of the literature. Oncol Lett. 2014;7(3):891-893.

3. Elder EE, Elder G, Larsson C. Pheochromocytoma and functional paraganglioma syndrome: no longer the $10 \%$ tumor. J Surg Oncol. 2005;89:193-201.

4. Kato H, Suzuki M, Mukai M, Aizawa S. Clinicopathological study of pheochromocytoma of the urinary bladder: immunohistochemical, flow cytometric and ultrastructural findings with review of the literature. Pathol Int 1999;49:1093-1099.

5. Zimmerman IJ, Biron RE, MacMahon HE. Pheochromocytoma of the urinary bladder. N Engl J Med 1953;249:25-26.

6. Nayyar R, Singh P, Gupta NP: Robotic management of pheochromocytoma of the vesicoureteric junction. JSLS. 2010;14:309-312.

7. Pandey R, Garg R, Roy K, Darlong V, Punj J, Kumar A. Perianesthetic management of the first robotic partial cystectomy in bladder pheochromocytoma. A case report. Minerva Anestesiol 2010;76:294-297.

8. Oderda M, Michelon F, Appendino M, et al. Primary bladder phaeochromocytoma diagnosed by a Vet. Scand J Urol Nephrol 2010;44:186-189.

9. Vesin C, Cadi P, Thony F, et al. A rare cause of complicated hypertension: urinary bladder paraganglioma. South Med J 2009;102:1173-1175.

10. Huang $Y$, Tian XJ, Ma LL. Pre-peritoneal laparoscopic partial cystectomy of the bladder pheochromocytoma. Chin Med J 2009;122:1234-1237.
11. Dhawan DR, Ganpule A, Muthu V, Desai MR. Laparoscopic management of calcified paraganglioma of bladder. J Urol. 2008;5:126-128.

12. Im SH, Kim NH. Thunderclap headache after micturition in bladder pheochromocytoma. Headache 2008;48:965967.

13. Siatelis A, Konstantinidis C, Volanis D, Leontara V, ThomaTsagli E, Delakas D. Pheochromocytoma of the urinary bladder: report of 2 cases and review of literature. Italian J Urol Nephrol 2008;60:137-140.

14. Kappers $M H$, van den Meiracker AH, Alwani RA, Kats E, Baggen MG. Paraganglioma of the urinary bladder. Neth J Med 2008;66:163-165.

15. Zwahlen D, Fishman PN, Honey J, Milosevic M, Tannock I. Malignant pheochromocytoma of the urinary bladder. Can J Urol 2007;14:3455-3457.

16. Bhalani SM, Casalino DD, Manvar AM: Paraganglioma of the bladder. J Urol 2011;186:279-280.

17. Kang SG, Kang SH, Choi H, Ko YH, Park HS, Cheon J. Robotassisted partial cystectomy of a bladder pheochromocytoma. Urol Int 2011;87:241-244.

18. Havekes B, Corssmit EP, Jansen JC, van der Mey AG, Vriends $\mathrm{AH}$, Romijn JA. Malignant paragangliomas associated with mutations in the succinate dehydrogenase $D$ gene. J Clin Endocrinol Metabol 2007;92:1245-1248.

19. Tsutsui A, Omoto K, Eto M, Naito $S$. Unsuspected pheochromocytoma of the urinary bladder in an 81-year-old woman. Hinyokika Kiyo 2006;52:577-579.

20. Bozbora A, Barbaros U, Erbil Y, Kilicarslan I, Yildizhan E, Ozarmagan S. Laparoscopic treatment of hypertension after micturition: Bladder pheochromocytoma. JSLS. 2006;10:263-266. 